\title{
An Improved Dual Sensor Summation Method with Application to Four-Component (4-C) Seafloor Seismic Data from the Niger Delta
}

\author{
Difference O. Ogagarue ${ }^{1} \&$ Joseph O. Ebeniro ${ }^{2}$ \\ ${ }^{1}$ Department of Earth Sciences, Federal University of Petroleum Resources, Effurun, Nigeria \\ ${ }^{2}$ Department of Geophysics, University of Port Harcourt, Nigeria \\ Correspondence: Difference O. Ogagarue, Department of Earth Sciences, Federal University of Petroleum \\ Resources, Effurun, Nigeria. E-mail: ogagarue.odeyovwi@fupre.edu.ng
}

Received: January 30, 2015

Accepted: March 24, 2015

Online Published: July 30, 2015

doi:10.5539/esr.v4n2p64

URL: http://dx.doi.org/10.5539/esr.v4n2p64

\begin{abstract}
Conventional towed streamer seismic acquisition has proven highly effective over the years for 3D subsurface imaging. However, in areas where water depths are too shallow or where there are too many obstacles for streamer operations, seabed seismic sensors have, in some cases, been employed as an alternative to image the subsurface. Apart from seabed seismic acquisition being extremely expensive compared to surface streamer, seabed seismic data are severely affected by receiver ghosts generated by water column reverberation. We present an improved method of summing the pressure (hydrophone data) and vertical geophone (geophone data) data to reduce the deleterious effects of ghosts and water column reverberations in a seabed seismic data acquired at water depths ranging from $21.1 \mathrm{~m}$ to $40.0 \mathrm{~m}$ in the Niger Delta. The method is based on the separation of up and down-going elastic wavefields from the geophone and hydrophone data, and provides an efficient noise rejection and amplitude preservation in the summed data.
\end{abstract}

Keywords: water column reverberations, seabed seismic, up and down-going elastic wavefields

\section{Introduction}

In seafloor seismic acquisition, sensor cables are laid on the seafloor while the shots are fired at some depths inside the water. The state-of-the-art sensors now have two sensors comprising a single hydrophone and a three-component geophone. The hydrophone measures pressure of the wavefield and since pressure is a scalar quantity, the measurement is not sensitive to the direction of the wavefield. The three-component (3-C) geophone measures particle velocity, and its measurement is sensitive to wavefield direction, recording different polarity depending on the direction of the wavefronts. The 3-C geophone is oriented in such a way that one component, called the Z-component, records the vertical component of the elastic wavefield or acceleration, and the other two components record the horizontal X-and Y-components of the wavefield. In this arrangement, the 3-C geophone records the full 3-D ground motion. Compressional waves are detected primarily by both the hydrophone and Z-component geophone while S-waves are primarily detected by the $\mathrm{X}$ - and Y-component geophones. This seafloor seismic acquisition is known as 4-C seismic acquisition, and has enabled geophysicists to gain more information about the subsurface than using vertical receivers only (Singh and Gautham, 1998).

When a seismic signal is reflected by a sub-surface boundary, the reflected wave travels towards the earth surface to the seafloor where it is recorded by both the hydrophone and 3-C geophone. The wavefield continues to travel upwards to the water surface, where it is internally reflected back into the water layer. When it reaches the seafloor, it is again recorded by the hydrophone and 3-C geophone. Some of the wavefield is again reflected back from the seafloor to the water surface and the cycle repeats, producing second and multiple arrivals of the original primary reflection, at time lags equal to the two-way traveltime through the water column (Hoffe et al., 2000). Thus, in seafloor seismic acquisition, the acquisition sensors (hydrophone and 3-C geophone) measure both the up-going and down-going elastic wavefields. Whereas the hydrophone responds to the pressure of the up-going and down-going wavefields due to the water column above the seafloor, the 3-C geophone responds to the velocity (particle) displacement of the up- and down-going elastic wavefields. The primary reflections constitute the up-going wafefields while all the subsequent water-column reverberations or receiver-side multiples constitute the down-going wavefields. 
One major challenge to the seismic imaging geophysicist is to migrate a dataset which is free from reverberations and multiples, as these have been known to destroy the quality of the imaged data. An effective way of separating the down-going from the up-going elastic wavefield thus constitutes the major challenge during pre-processing of the data. One way of mitigating this challenge is dual sensor summation, in which the hydrophone signals are summed with the vertical geophone signals to suppress the receiver-side multiples and reverberations. In this paper, we present a dual sensor summation method which successfully separated the upand down-going wavefields in a 4-C dataset obtained from the Niger Delta shallow marine environment with average water depth of $30.6 \mathrm{~m}$, resulting in a dataset effectively suppressed of (receiver-side) multiples and reverberations.

\section{Review of Wave Field Separation Methods}

Several methods are in the literature on the separation of the up-going and down-going elastic wavefields in seafloor seismic data by a combination of the hydrophone record (wavefield pressure, $\mathrm{H}$ ) and a scaled version of the vertical component geophone record $(\mathrm{G})$ for the removal of reverberations and multiples from the seismic record (Loewenthal et al., 1985; Barr and Sanders, 1989; Draggoset and Barr, 1994; Paffenholz and Barr, 1995; Ball and Corrigan, 1996; Soubaras, 1996; Bale, 1998). Most of these methods require the reflection coefficient at the seafloor to be accurately estimated to have success in multiple suppression.

Hoffe et al., 2000, presented a simple and robust method of dual-sensor summation that does not require an estimate of the seafloor reflection coefficient for the suppression of the receiver-side water-column multiples. Their approach computes time-variant scalar traces for every hydrophone-geophone trace pair, which forces the amplitude of the geophone traces to become near equal to that of the hydrophone traces. When summed, receiver-side multiple cancellation is achieved due to the opposite polarity of the hydrophone-geophone trace pairs at the ocean floor. Saleh and Daniel, 2005, applied this method to OBC dataset from the Arabian Gulf and successfully suppressed water-column receiver-side multiples generated at the receiver side. They, however, applied a median filter to the scalar traces calculated which improved the summation process and eliminated noise.

In this paper, we present an approach which does not also require estimation of the seafloor reflection coefficient, but essentially utilizes the down-going elastic wavefield to improve the image quality. Firstly, our approach utilizes the wavefield decomposition technology to separate the hydrophone and vertical geophone data into up-going and down-going waves, following the Hoffe et. al., 2000, procedure. Thereafter, bulk and time-variant scalars are calculated and applied before summing up the elastic wavefields.

\section{Materials and Methods}

\subsection{Dataset}

The dataset used for this study consists of (4-C) seafloor seismic data acquired over water depths ranging from $21.1 \mathrm{~m}$ to $40 \mathrm{~m}$ in the Niger Delta. The X-and Y-component records of the geophones were discarded, making the dataset a PP (2-C) dataset. Prior to the summation of the hydrophone and vertical geophone, coherent noise attenuation was carried out on the individual dataset to attenuate the swell noise and scholte wave-type noise found in the data. It was observed that the vertical geophone data was more noisy than the hydrophone data, therefore, several modifications were made to the parameterization to attack the vertical geophone noise.

\subsection{Methodology}

The sequence of reverberations for seismic arrivals trapped within the water column is shown in Figure 1 (Hoffe et al., 2000).

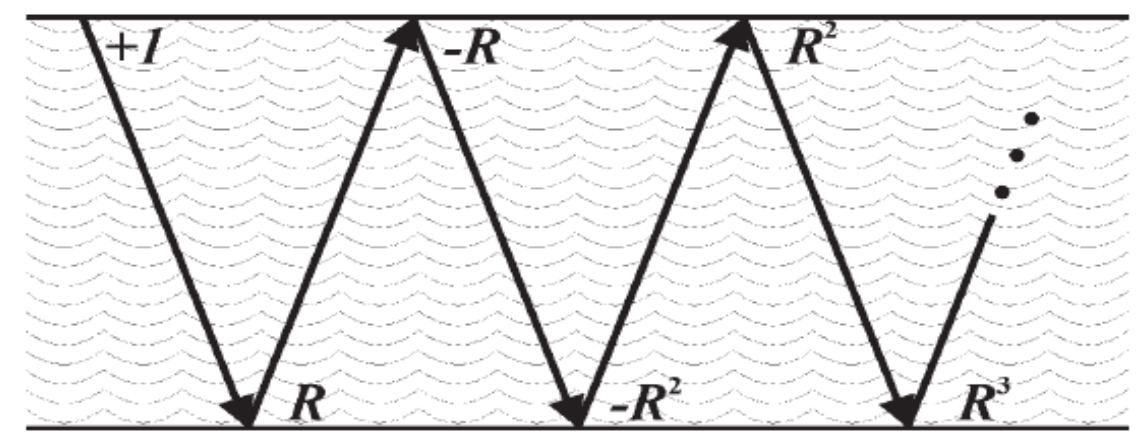

Figure 1. Reverberatory sequence for seismic arrivals trapped within the water column (Hoffe et al., 2000) 
If we consider vertical raypath in the water column, using the 1-D wave equation, Loewenthal et al., 1985 show that the pressure wavefield is the sum of the up-going and down-going wavefields, and the scaled velocity of displacement of the wavefield is the difference between the up-going and down-going wavefields. Since the hydrophone records the pressure wavefield and vertical geophone records the velocity of displacement of the wavefield, we can write the Loewenthal et al. (1985) equations in terms of the hydrophone and vertical geophone data as follows:

$$
\mathrm{H}=\mathrm{U}+\mathrm{D}
$$

and the velocity of displacement of the wavefield is given by:

$$
(\rho \mathrm{c}) \mathrm{V}=\mathrm{G}=\mathrm{U}-\mathrm{D}
$$

where $\mathrm{U}$ and $\mathrm{D}$ are the up-going and down-going elastic waves, $\rho$ and $\mathrm{c}$ are density of water and $\mathrm{P}$-wave velocity in water, respectively, and $\mathrm{G}$ is scaled vertical geophone data.

At the ocean bottom, the $\mathrm{Z}$ transform of the down-going wave is given by:

$$
\mathrm{D}(\mathrm{z})=1-\mathrm{Rz}+\mathrm{R}^{2} \mathrm{z}^{2}+\ldots \ldots \ldots \cdot \cdots=\frac{1}{1+\mathrm{Rz}}
$$

and that for the up-going wave is given by:

$$
\mathrm{U}(\mathrm{z})=\mathrm{R}-\mathrm{R}^{2} \mathrm{z}+\mathrm{R}^{3} \mathrm{z}^{2}+\ldots \ldots \cdot=\frac{\mathrm{R}}{1+\mathrm{Rz}}
$$

The $\mathrm{Z}$ transforms of $\mathrm{H}$ and $\mathrm{G}$ are given by:

$$
H(z)=U(z)+D(z)=\frac{1}{1+R z}(1+R)
$$

and

$$
\mathrm{G}(\mathrm{z})=\mathrm{U}(\mathrm{z})-\mathrm{D}(\mathrm{z})=\frac{\mathrm{R}-1}{1+\mathrm{Rz}}
$$

Combining Equations (5) and (6), we see that:

$$
\mathrm{G}(\mathrm{z})+\left[\frac{1-\mathrm{R}}{1+\mathrm{R}}\right] \mathrm{H}(\mathrm{z})=0
$$

Equation (7) shows that a summation of the scaled vertical geophone record $(G(z)$ which measures the velocity of particle displacement and a scaled version of the hydrophone record which measures the wavefield pressure will remove all receiver-side ghosts and reverberations.

In implementing the dual sensor summation technique presented here, the hydrophone and vertical geophone datasets were initially denoised and individually sorted into common receiver domain. The original RMS amplitudes of the hydrophone records are commonly an order of magnitude greater than those of the vertical geophone records (Fig. 2), and to calibrate the hydrophone records thereby matching their RMS amplitudes to those of the vertical geophone records, the hydrophone traces were stacked and transformed into the frequency domain, where they were differentiated using the Fourier transform:

$$
F_{(\omega)}=\int_{-\infty}^{\infty} f(t) \exp (-i \omega t) d t-\int_{-\infty}^{\infty} f(t-1) \exp (-i \omega t) d t
$$

where,

$$
\begin{aligned}
& \mathrm{f}(\mathrm{t})=\operatorname{trace}_{(\mathrm{i})} \\
& \mathrm{f}(\mathrm{t}-1)=\operatorname{trace}_{(\mathrm{i}-1)} .
\end{aligned}
$$

As shown in Equation 8, the differentiation was implemented by simply multiplying each frequency band in the hydrophone data by $2 \pi \mathrm{f} \sqrt{-i}$, resulting in trace $(i)=$ trace $_{(i)}$ - trace $_{(i-1)}$. 


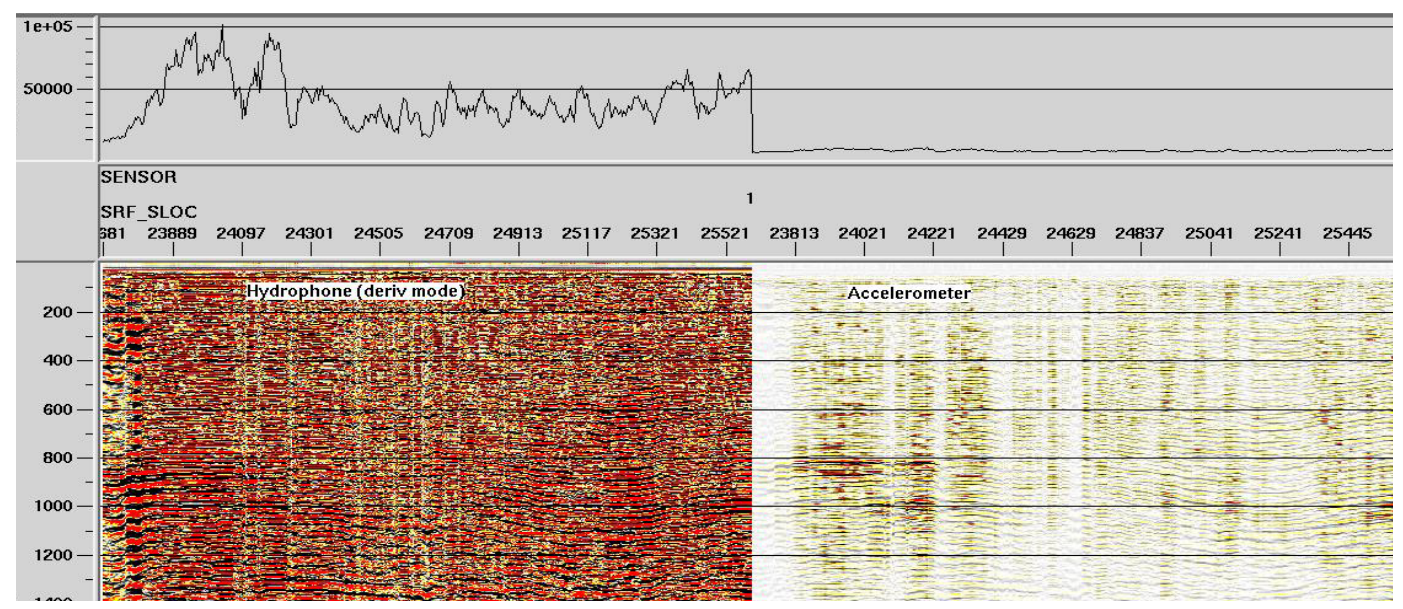

Figure 2. Original RMS amplitudes of Hydrophone and Vertical Geophone data

An approach was thereafter employed in which incremental bulk scalars were statistically derived and applied to the RMS amplitudes of the hydrophone data in the entire region (Fig. 3). The scalar which gave the best RMS amplitude match for all lines was chosen and was finally applied to scale down the hydrophone RMS amplitudes to match the Accelerometer dataset. This process effectively calibrated the RMS amplitudes of the primaries of the hydrophone data to match those of the vertical geophone data.

\section{Hydrophone RMS amplitude scaling te st}

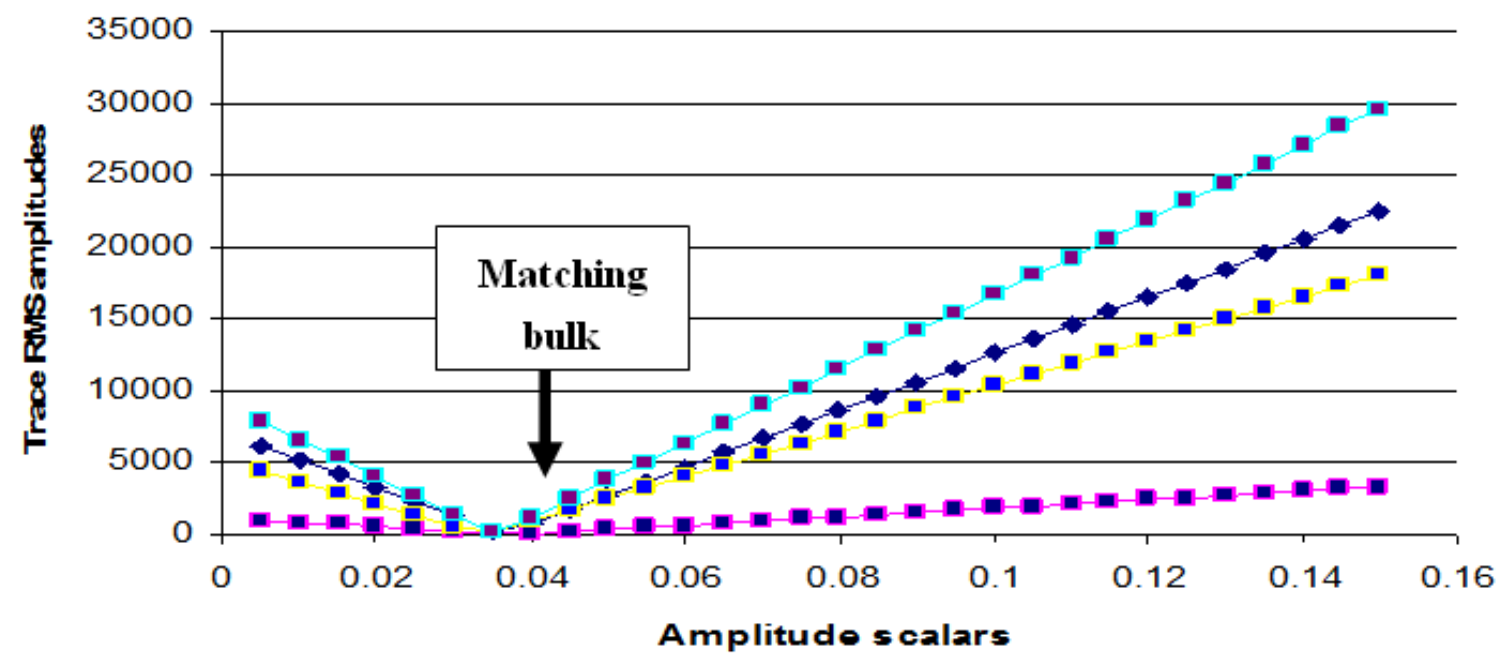

Figure 3. Calibration factors for bulk scalar application to hydrophone data. Thirty (30) scalar panels ranging from 0.005 to 0.15 were tested over four (4) receiver lines (RL). RMS values for the RLines in the Figure converged best at panel 7. The scalar value at this panel was used to scale the hydrophone data

Next, a stack of the vertical geophone and hydrophone pair of traces was created and the elastic wavefields were decomposed into up-going and down-going waves using the relations:

$$
\begin{aligned}
& \text { Up - going wavefield }=\mathrm{H}_{\mathrm{bs}}+\mathrm{G}_{\mathrm{ds}} \\
& \text { Down - going wavefield }=\mathrm{H}_{\mathrm{bs}}-\mathrm{G}_{\mathrm{ds}}
\end{aligned}
$$

where $\mathrm{H}_{\mathrm{bs}}$ and $\mathrm{G}_{\mathrm{ds}}$

are respectively the bulk-scaled hydrophone record and vertical geophone record with a dynamic scalar applied. To derive the dynamic scalar, ds, we cross-correlated the up-going and down-going elastic wavefields in the 
manner:

$$
\begin{aligned}
& \text { Up - going wavefield }=\mathrm{H}_{\mathrm{bs}}+\mathrm{G} \\
& \text { Down - going wavefield }=\mathrm{H}_{\mathrm{bs}}-\mathrm{G}
\end{aligned}
$$

where $G$ is original vertical geophone record, over a range of possible scalar values. The dynamic scalar value, $\mathrm{ds}$, which gave the lowest cross-correlation coefficient between the up and down going wavefields was then selected and applied to the vertical geophone records to obtain the desired up-going and down-going wavefields (Equation 9). Application of the dynamic scalar to the vertical geophone records effectively calibrated the ghost reflections of the vertical geophone data by matching their RMS amplitudes to those of the hydrophone ghosts. Calibration of the vertical geophone ghosts by application of the dynamic scalar somewhat boosts the RMS amplitudes of the primary reflections of the vertical geophone data. To minimize this effect, the RMS amplitudes were normalized using the relation:

$$
\begin{gathered}
\text { Up - going elastic wavefield }=\left[\frac{\mathrm{H}_{\mathrm{bs}}+\mathrm{G}_{\mathrm{ds}}}{1+\mathrm{ds}}\right] \\
\text { Down - going elastic wavefield }=\left[\frac{\mathrm{H}_{\mathrm{bs}}-\mathrm{G}_{\mathrm{ds}}}{2}\right]
\end{gathered}
$$

\section{Results and Discussion}

\subsection{Results}

RMS amplitudes of primary reflections of the hydrophone data, calibrated to match those of the vertical geophone data by application of the bulk scalar to the hydrophone data is shown in Fig. 4, and Fig. 5 shows a pair of hydrophone and vertical geophone traces before and after the calibration.

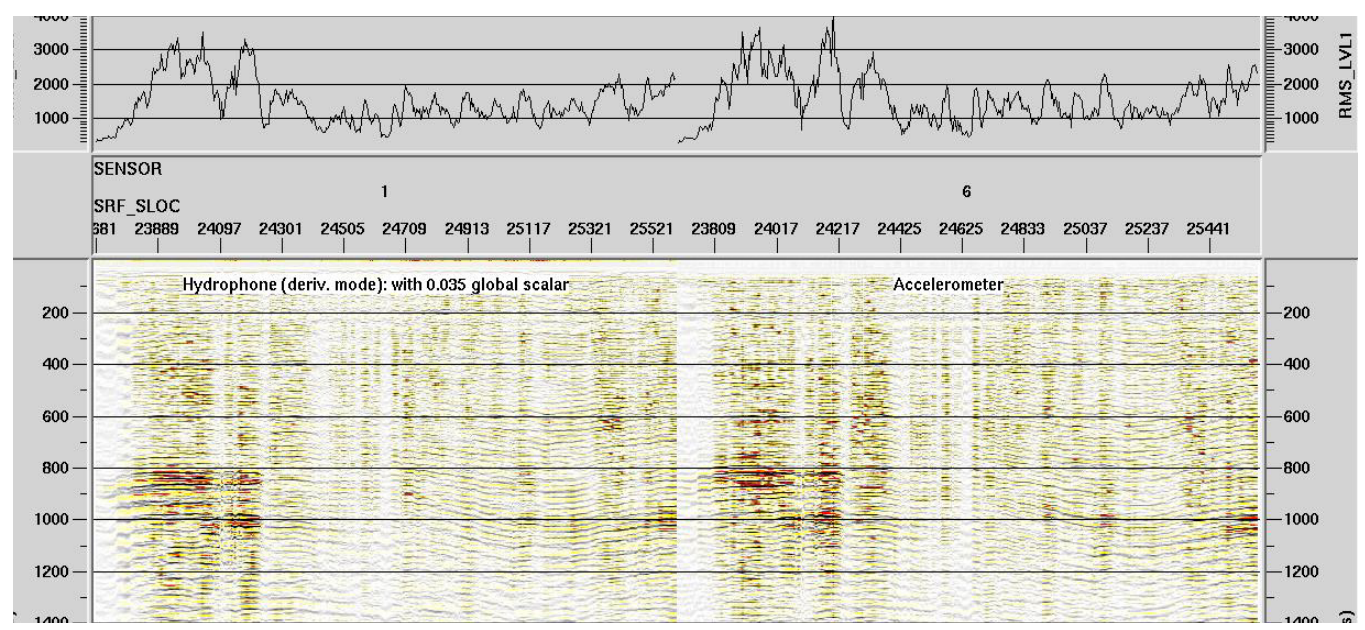

Figure 4. RMS amplitudes after application of bulk scalar to hydrophone data to match the vertical geophone RMS amplitudes

The calibration effectively matched the RMS amplitudes of the primaries of the hydrophone record to those of the vertical geophone record (Fig. 4). The amplitudes of the receiver-side ghost and reverberations of the hydrophone data were also significantly scaled, but did not match those of the vertical geophone data. Therefore, summation of the sensors at this stage would greatly enhance the primaries, but would not lead to cancellation of the receiver-side ghosts. Application of the dynamic scalar to the vertical geophone data was aimed at matching the amplitudes of the ghosts of the geophone data to those of the hydrophone data after normalization of the amplitudes. The applied scalar was effective in matching the amplitudes of the receiver-side ghosts and reverberations of the two datasets, and these completely cancelled by the summation process. 


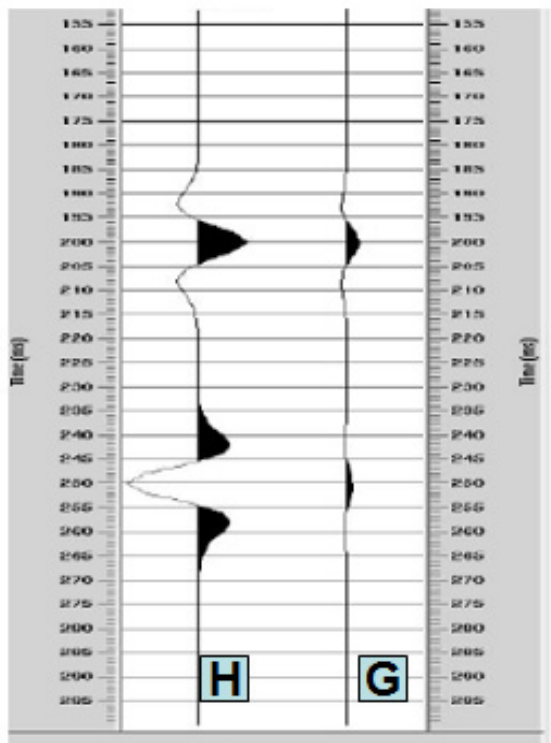

(a)

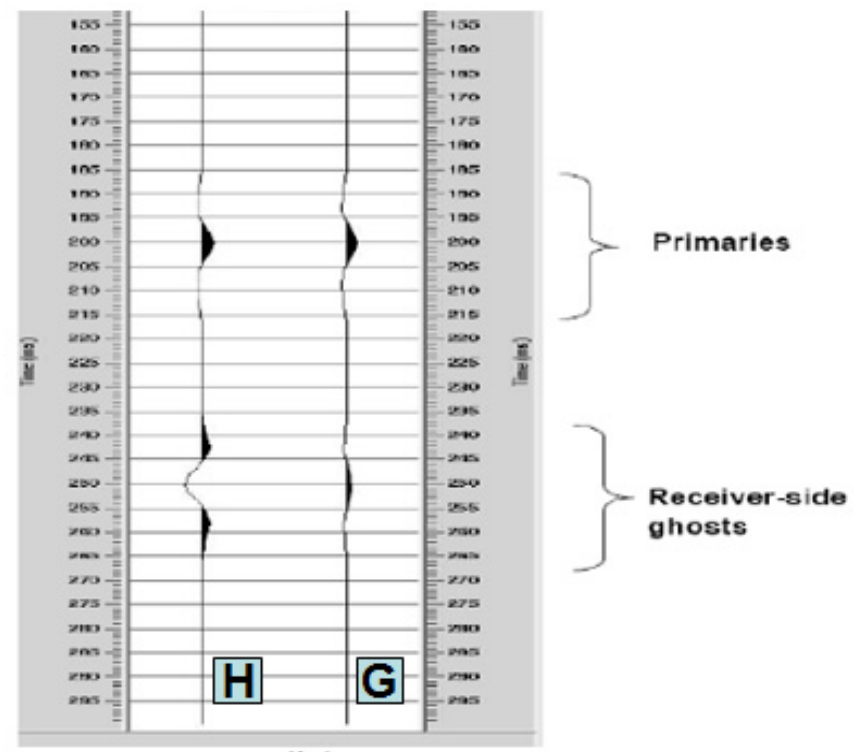

(b)

Figure 5. Trace amplitudes of hydrophone and vertical geophone data. (a) Original amplitudes, (b) amplitudes after application of bulk scalar to hydrophone

\subsection{Discussion}

Since hydrophone measures the wavefield pressure (up- and down-going waves) due to the water-column, and pressure is a scalar quantity which is not sensitive to wave direction, the polarity of the recorded data is the same for the up- and down-going waves for the hydrophone. The vertical phone measures particle velocity of the wave motion, which is sensitive to the wavefield direction. As a result, the vertical geophone measures opposite polarity for the up-going (+ve polarity) and down-going (-ve polarity) elastic wavefields (Fig. 6).
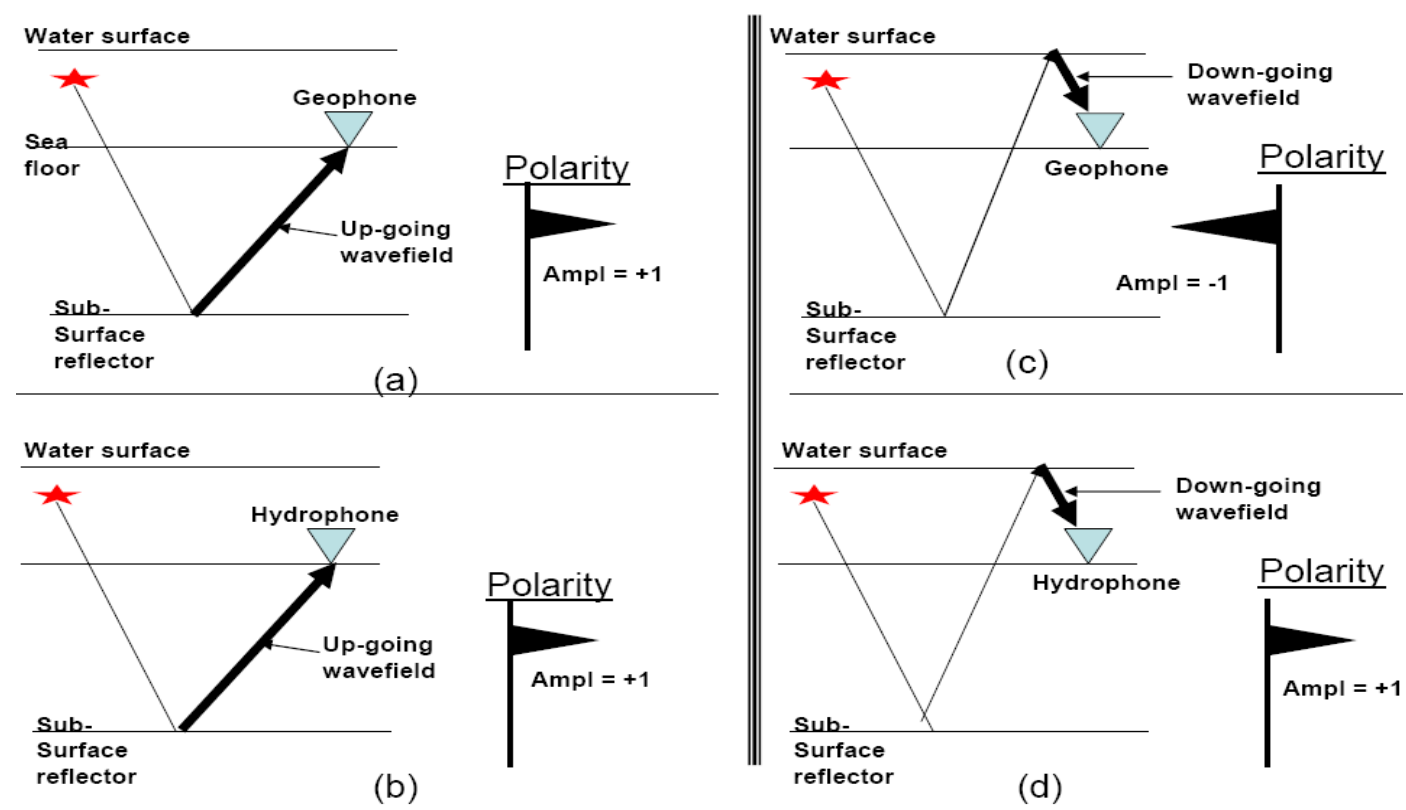

(b)

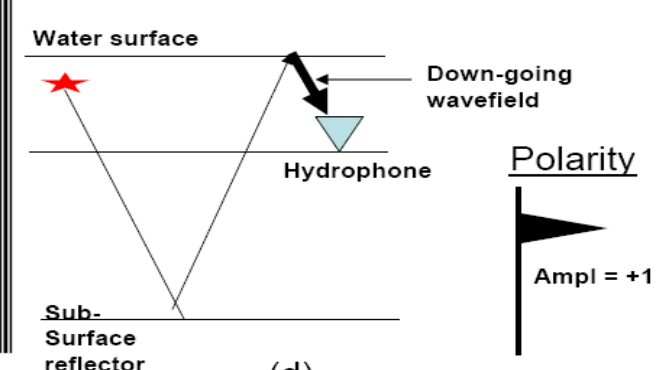

(d)

Figure 6. Polarity of up- and down-going waves recorded by the vertical geophone and hydrophone . Up- and down-going wavefields recorded by the hydrophone have the same polarity (a and d), but the polarity is opposite for the up- and down-going wavefields recorded by the vertical geophone (a and c).

The goal of dual sensor summation is to fill in the spectral notches present in the hydrophone and vertical geophone data, producing a dataset having receiver-side reverberations which would be cancelled upon 
summation. Figure 7 and Figure 8 show a gather of the hydrophone, vertical geophone and the corresponding summed dataset and their stacks, respectively. The frequency spectrum of the stacked datasets before and after the summation is shown in Figure 9.

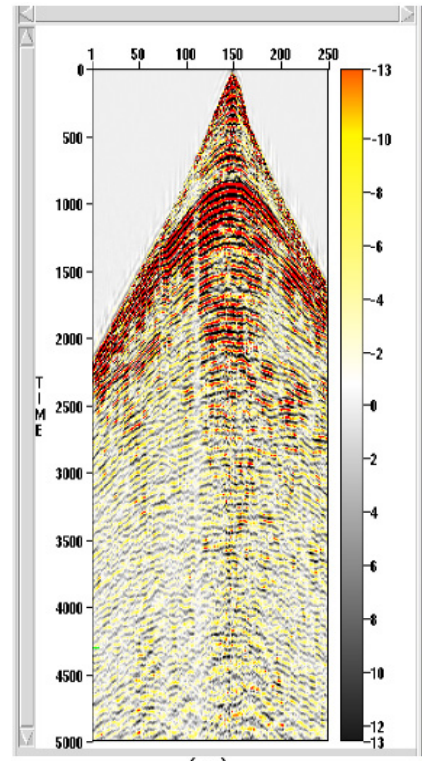

(a)

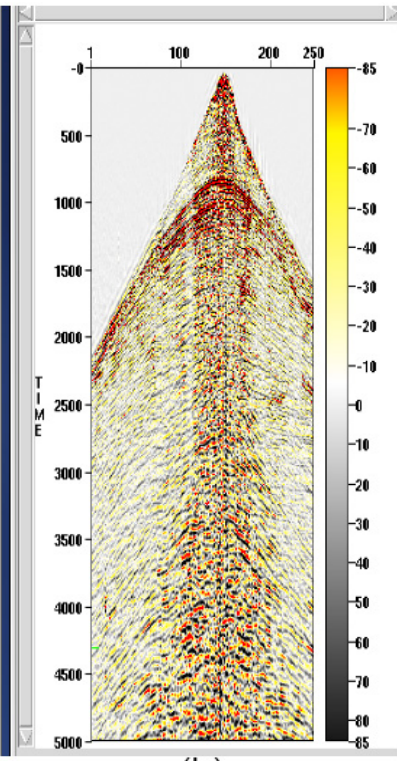

(b)

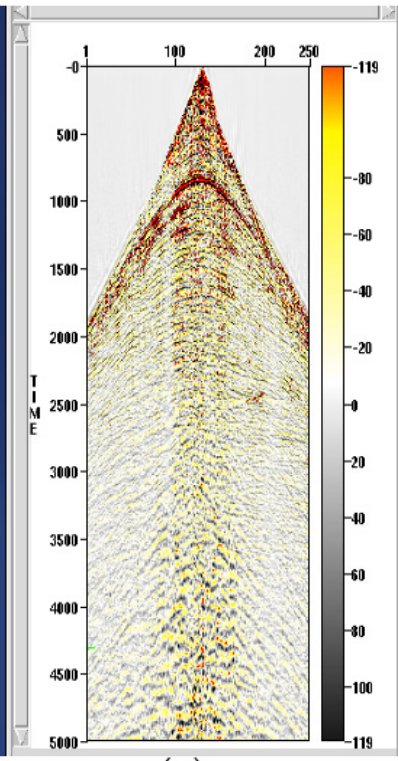

(c)

Figure 7. Common receiver gathers of (a) hydrophone (b) vertical geophone and (c) HG sum gathers. Multiples are considerably reduced in (c).

The hydrophone gather is contaminated with multiples, while the summed gather is cleaned up considerably. Multiples are less prevalent in the vertical geophone gather than the hydrophone gather. The stacks also show that the summed dataset contains less of multiples than the vertical geophone and hydrophone data. Fig. 9 shows that the notches present in the recorded data before the summation are effectively filled after the summation. This further proves the success of the dual sensor summation method presented.
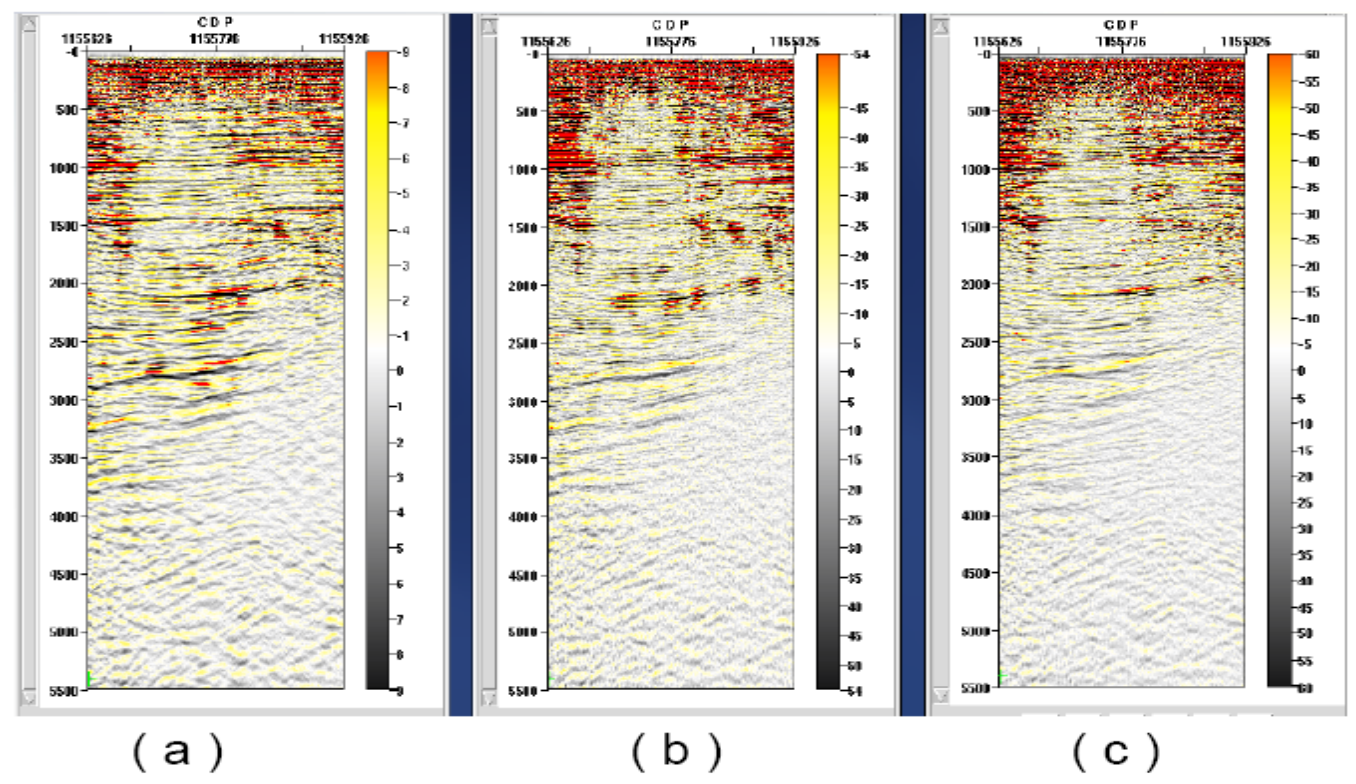

Figure 8. Common receiver stacks (CRS) of hydrophone (a), vertical geophone (b) and HG sum (c) datasets 


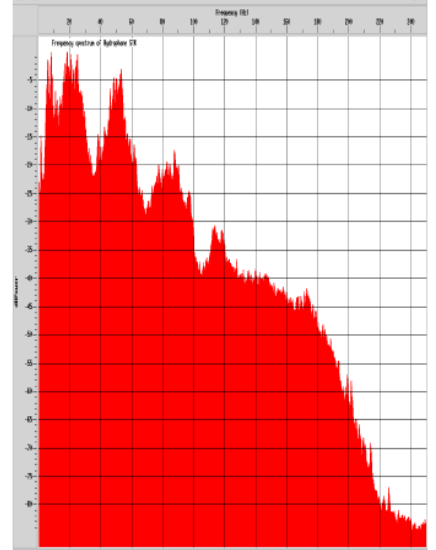

(a)

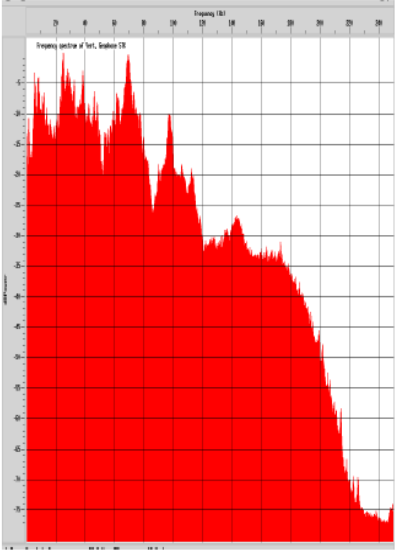

(b)

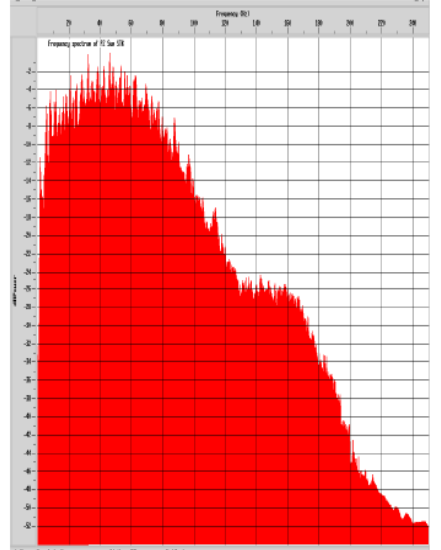

(c)

Figure 9. Frequency spectrum of (a) hydrophone stack, (b) vertical geophone stack, and (c) HG sum stack. The notches are prevalent in the hydrophone spectrum than vertical geophone spectrum, but are effectively filled in the HG sum spectrum

As is shown in the gathers and stacks, we still have remnant of reverberations in the summed dataset, but these have been reduced considerably. It is worthy to mention that the dual sensor summation method presented in this paper does not attack the source-related multiples. The shooting geometry of seafloor seismic data acquisition results in source-related ghosts and multiples in the data (Fig. 10), since the shot is taken at some depth inside the water layer. Some energy would first impinge on the water surface directly from the source (ghost energy), and this would be reflected into the subsurface, thereby becoming secondary signals. The primary signals are those that propagate directly from the source into the subsurface and are picked up by the sensors after reflection from the subsurface boundaries. This summation method is not intended to address the source-related multiples and ghosts. These must be attenuated using other techniques.

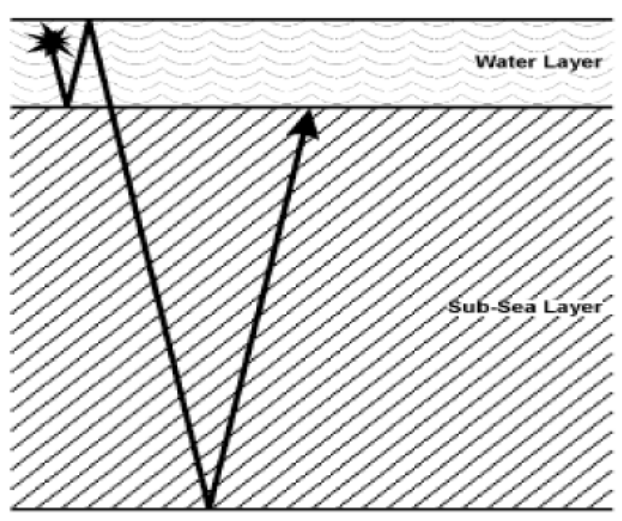

(a)

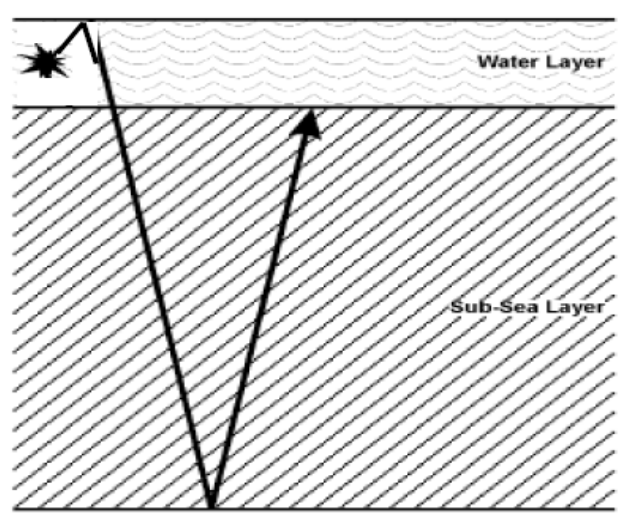

(b)

Figure 10. (a) Source-side water reverberation dependent on the depth of water layer, and (b) Source-side ghost dependent on the depth of the source

Autocorrelations of the hydrophone, vertical geophone and summed dataset over the time window 200ms $3500 \mathrm{~ms}$ are shown in Figure 11. Autocorrelations show the degree of repetitiveness in a dataset. The degree of ringing is much reduced in the HG sum dataset compared to the hydrophone and vertical geophone. This confirms that the HG summation procedure was effective in cleaning up the data, producing a dataset that would be more representative of the subsurface. 


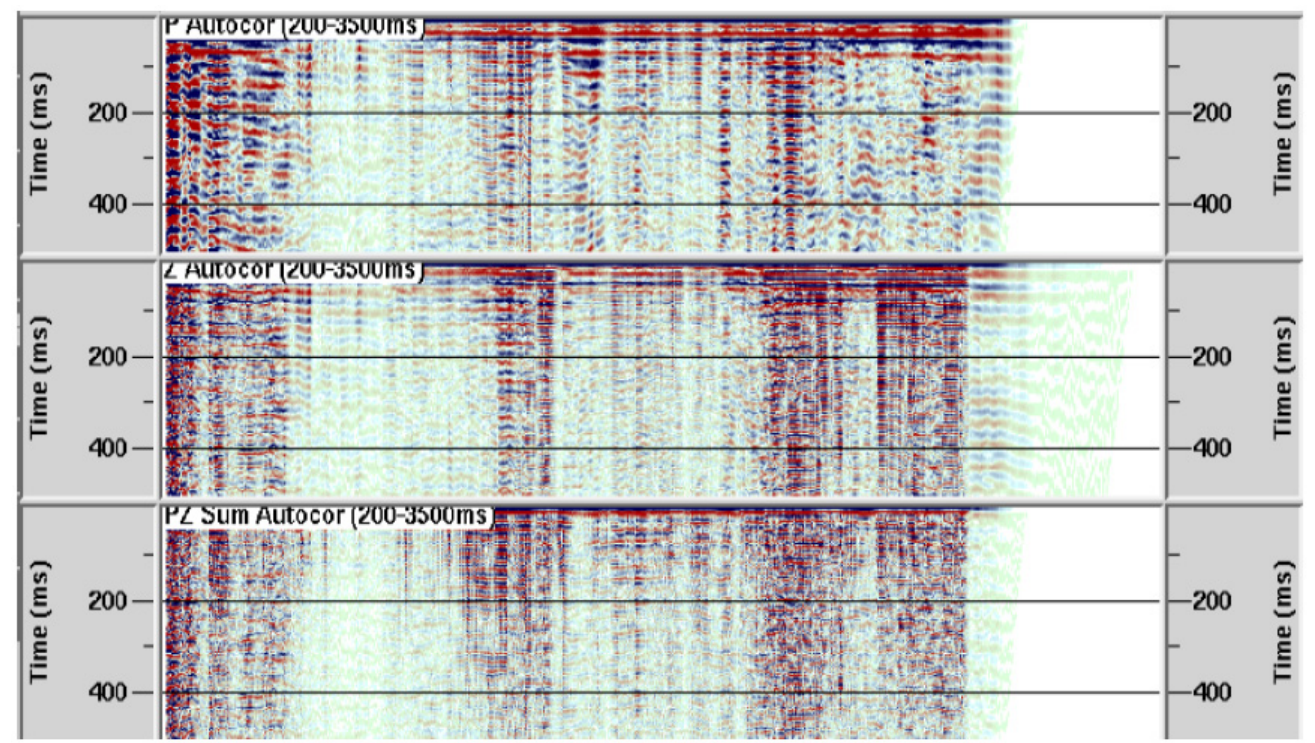

Figure 11. Autocorrelations of the hydrophone $(\mathrm{H})$, vertical geophone $(\mathrm{G})$ and summed $(\mathrm{HG})$ traces. The water-column reverberations are less prevalent in the HG sum dataset

To further check the effectiveness of the multiple suppression using this technique, we produced a map of the hydrophone-vertical geophone (HG) summation scalars (Fig. 12) to investigate receiver coupling in the seafloor. Any receiver that is not properly coupled with the seafloor will show an anomalous scalar value (Saleh and Daniel, 2005). The map shows that the scalars are fairly consistent on a global scale. A few receivers that had high scalar values were due to bad shots. These were edited for a re-computation of the scalars.

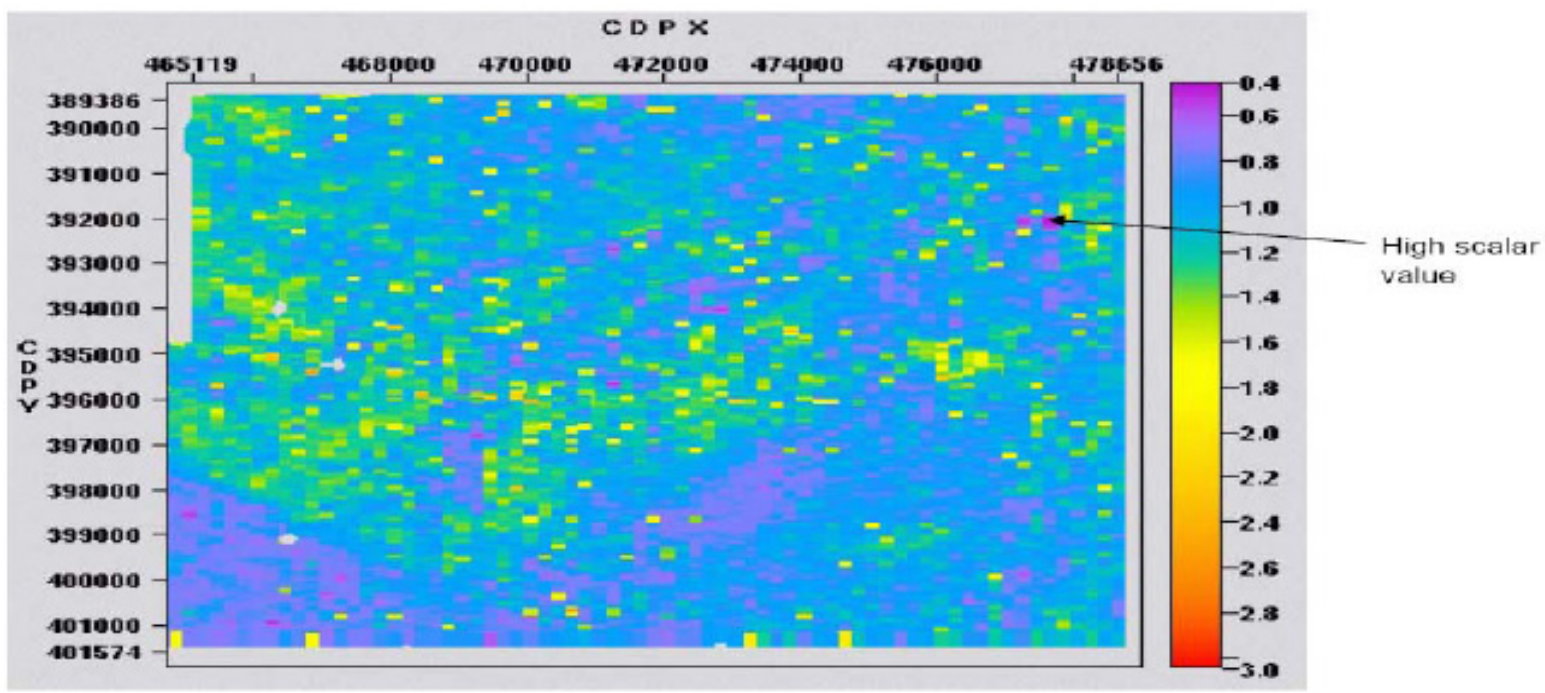

Figure 12. HG summation scalars

\section{Conclusion}

We applied a dual summation method based on application of bulk and dynamic scalars to an OBC data from the Niger Delta and successfully suppressed water-column reverberation and multiples generated at the receiver side. The method applied does not require an estimation of the seafloor reflection coefficient, but utilizes the wavefield decomposition methodology to separate the hydrophone and vertical geophone data into up-going and down-going wavefields. The up-going wavefield is the normalized sum of the hydrophone (on which a bulk scalar has been calculated and applied) and scaled vertical geophone. The down-going wavefield is the normalized difference of the hydrophone and scaled vertical geophone. The summation relied on the fact that the receiver-side ghosts cancel upon summation of the up- and down-going fields after application of the scalars. 
The method accounts for local variations in seafloor reflectivity, and was efficient in suppressing the water-column reverberations and multiples generated at the receiver side.

\section{References}

Bale, R. (1998). Plane wave de-ghosting of hydrophone and geophone OBC data. $68^{\text {th }}$ Ann. Internat. Mtg., Soc. Expl. Geophys., Expanded Abstracts, 730-733.

Ball, V., \& Corrigan, D. (1996). Dual-sensor summation of noisy ocean-bottom data $66^{\text {th }}$ Ann. Internat. Mtg., Soc. Expl. Geophys., Expanded Abstracts: 28-31.

Barr, F. J., \& Sanders, J. I. (1989). Attenuation of water-column reverberations using pressure and velocity detectors in a water-bottom cable. 59 ${ }^{\text {th }}$ Ann. Internat. Mtg:, Soc. Expl. Geophys., Expanded Abstracts, 653-656.

Draggoset, B., \& Barr, F. J. (1994). Ocean-bottom cable dual-sensor scaling. $64^{\text {th }}$ Ann. Internat. Mtg., Soc. Expl. Geophys., Expanded Abstracts, 857-860.

Hoffe, B. H., Lines, L. H., \& Cary, P. W. (2000). Applications of OBC Recording. The Leading Edge, 19 (4).

Loewenthal, D., Lee, S. S., \& Gardner, G. H. (1985). Deterministic estimation of wavelet using impedance type techniques, Geophys. Prosp., 33, 956-969.

Saleh, M. A., \& Daniel, N. A. (2005). A robust dual sensor summation method Application to OBC data from the Arabian Gulf. Evolving Geophysics Through Innovation, Canadian Society of Exploration Geophysicists, National Convention, p. 389-392.

Paffenholz, J., \& Barr, F. J. (1995). An improved method for determining water bottom reflectivities from dual-sensor ocean bottom cable data. $65^{\text {th }}$ Ann. Internat. Mtg., Soc. Expl. Geophys., Expanded Abstracts, 987-989.

Singh, V., \& Gautam, S. (1998). Developments in Offshore Data Acquisition Technology: An Overview. GEOHORIZONS, 3(2), 1-11.

Soubaras, R. (1996). Ocean-bottom hydrophone and geophone processing. $66^{\text {th }}$ Ann Internat. Mtg., Soc. Expl. Geophys., Expanded Abstracts, 24-27.

\section{Copyrights}

Copyright for this article is retained by the author(s), with first publication rights granted to the journal.

This is an open-access article distributed under the terms and conditions of the Creative Commons Attribution license (http://creativecommons.org/licenses/by/3.0/). 